\title{
Ácidos Graxos Poli-insaturados Ômega-3: saúde cardiovascular e sustentabilidade ambiental ${ }^{1}$
}

\author{
Julicristie Machado de Oliveira ${ }^{2}$, Liania Alves Luzia ${ }^{3}$, \\ Patrícia Helen de Carvalho Rondó ${ }^{4}$
}

Os Ácidos Graxos Poli-insaturados Ômega-3 (n-3 PUFA) se referem a um conjunto de lipídios, incluindo os Ácidos Alfa-linolênico (ALA), Eicosapentaenóico (EPA) e Docoxahexaenóico (DHA). Estudos que tratam do papel do n-3 PUFA são abundantes e seu papel cardioprotetor está bem discutido na literatura, porém seus efeitos são mais evidentes na prevenção secundária, isto é, em populações com alguma alteração cardiovascular prévia. Apesar da ausência de fortes evidências em relação à prevenção primária, recomenda-se o consumo de peixe duas vezes por semana, além de outros alimentos fontes do ácido graxo, para pessoas sem doença cardiovascular diagnosticada. Equacionar as recomendações para a saúde e a possibilidade ambiental de atendêlas constitui-se em um grande desafio. As reservas de peixe têm sofrido rápido declínio nos últimos anos devido a seu caráter essencialmente extrativista, pois até mesmo a aquicultura envolve a utilização de pequenos peixes selvagens para a produção de ração. A utilização do óleo de microalgas, como fonte de n-3 PUFA para a aquicultura e para o consumo humano, pode ser uma alternativa, mas deve-se avaliar seu uso com responsabilidade para que haja preservação ambiental e garantia da segurança alimentar e nutricional.

Palavras-chave: ácidos graxos ômega-3, ácido alfa-linolênico, ácido docosahexaenóico, ácido eicosapentaenóico, suplementos dietéticos, doenças cardiovasculares, sustentabilidade, Segurança Alimentar e Nutricional.

\section{Omega-3 polyunsaturated fatty acids: cardiovascular health and environmental sustainability}

Omega-3 polyunsaturated fatty acids (n-3 PUFA) refer to a set of lipids, such as Alpha-linolenic Acid (ALA), Eicosapentaenoic Acid (EPA) and Docoxahexaenoic (EPA). The effects of n-3 PUFA on secondary prevention of cardiovascular diseases are well discussed in the literature. Despite the absence of strong evidence for primary prevention, the consumption of fish twice a week, and other sources of n-3 PUFA, is recommended for healthy subjects. To balance the recommendations with the environmental sustainability to meet them is a big challenge. Fish stocks have suffered a sharp decline in recent years. Even the aquaculture involves the use of small wild fish for feed production. Thus, the use of microalgae oil as a source of n-3 PUFA for aquaculture and human

\footnotetext{
${ }^{1}$ Financiamento FAPESP: Processos 2008/55532-9 e 2008/50970-8.

2 Profa. Dra. Faculdade de Ciências Aplicadas (FCA), Universidade Estadual de Campinas, UNICAMP. Correspondência: Faculdade de Ciências Aplicadas (FCA), UNICAMP. Rua Pedro Zaccaria, 1300, Jardim Santa Luiza, CEP 1348-350, Limeira, SP. E-mail: julicristie.oliveira@fca.unicamp.br

3 Doutora em Saúde Pública, Departamentode Nutrição, Faculdade de Saúde Pública (FSP), Universidade de São Paulo (USP).

${ }^{4}$ Profa. Titular, Departamento de Nutrição, Faculdade de Saúde Pública (FSP), Universidade de São Paulo (USP).
} 
consumption can be an alternative, but its use should be assessed with responsibly to guarantee environmental preservation and food security.

Key-words: omega-3 fatty acids, alpha-linolenic acid, docosahexaenoic acids, eicosapentaenoic acid, dietary supplements, cardiovascular diseases, sustainability, food security.

\section{INTRODUÇÃO}

Ácidos Graxos Poli-insaturados Ômega-3 (n-3 PUFA) constituem um termo genérico que se refere a um conjunto de lipídios que possuem uma insaturação, ou dupla ligação, na posição n-3 das cadeias hidrocarbonadas. Como os mamíferos não apresentam enzimas que possuem habilidade de inserir dupla ligação nessa posição, esses ácidos graxos são considerados essenciais. São exemplos de n-3 PUFA os Ácidos Graxos Alfa-linolênico (ALA), Docosahexaenóico (DHA) e Eicosapentaenóico (EPA) [1].

O papel cardioprotetor dos n-3 PUFA, presentes em peixe ou em óleo de peixe, é muito discutido na literatura científica. Os possíveis mecanismos pelos quais esses ácidos graxos reduzem o risco de eventos cardiovasculares incluem: promoção do relaxamento endotelial, desaceleração da formação de placas de ateroma, ação anti-inflamatória e antitrombogênica; redução da expressão das moléculas de adesão, da agregação de plaquetas, de triglicérides e da susceptibilidade à arritmia ventricular [2,3].

O objetivo do presente artigo é descrever o teor e composição de n-3 PUFA em alguns alimentos e suplementos, bem como discutir seus efeitos na saúde cardiovascular e questões conducentes à sustentabilidade.

\section{Alimentos fontes e composição dos ácidos graxos}

Muitos alimentos ricos em n-3 PUFA também apresentam alto teor de Ácidos Graxos Poliinsaturados Ômega-6 (n-6 PUFA). Como a razão n6/n-3 parece ser mais importante que a quantidade absoluta de n-3 na dieta, a redução do consumo de n-6 e aumento do consumo de n-3 é indicada [4]. Sendo assim, serão descritos como fontes somente os alimentos que apresentam teores mais elevados de n-3 PUFA quando comparados aos teores de n-6 PUFA e que contribuem com ao menos $10 \%$ da Ingestão Adequada (AI) de acordo com recomendações nutricionais do Institute of Medicine ${ }^{[1]}$ (Quadro 1).

São fontes de n-3 PUFA alimentos de origem vegetal e animal. Os alimentos de origem vegetal terrestres são ricos em ALA, que no organismo humano pode ser convertido a EPA e DHA. A maior parte dos alimentos de origem marinha (vegetal ou animal) são fontes de ambos, porém apresentam predomínio de DHA na composição. O Quadro 2 apresenta exemplos de alimentos fonte e composição dos ácidos graxos, ácido graxo predominante e teor total de acordo com uma porção usual de consumo.

\begin{tabular}{|lc|}
\hline Faixa Etária/Estado Fisiológico & $\begin{array}{c}\text { AI } \\
\text { (mg/dia) }\end{array}$ \\
\hline Crianças no primeiro ano de vida & \\
$0-12$ meses & 500 \\
Crianças & \\
$1-3$ anos & 700 \\
$4-8$ anos & 900 \\
Sexo masculino & \\
$9-13$ anos & 1200 \\
$\geq 14$ anos & 1600 \\
Sexo feminino & \\
9-13 anos & 1000 \\
$\geq 14$ anos & 1100 \\
Gravidez (qualquer idade) & 1400 \\
Lactação (qualquer idade) & 1300 \\
\hline Fonte: Institute of Medicine ${ }^{1]}$ & \\
\hline
\end{tabular}

Quadro 1. Ingestão Adequada (AI) de n-3 PUFA. 


\begin{tabular}{|c|c|c|c|c|c|}
\hline \multirow{2}{*}{ Alimento } & \multicolumn{4}{|c|}{$\begin{array}{c}\text { Composição de n-3 PUFA por } \\
\text { porção }(\mathrm{mg})\end{array}$} & \multirow{2}{*}{$\begin{array}{c}\text { Teor de n-3 PUFA (mg) de acordo } \\
\text { com a porção }\end{array}$} \\
\hline & $\mathrm{ALA}^{1}$ & $\mathrm{EPA}^{2}$ & $\mathrm{DHA}^{3}$ & Outros & \\
\hline \multicolumn{6}{|l|}{ Origem vegetal $*$} \\
\hline Semente de linhaça & 2350 & - & - & - & 1 colher de sopa $(10,3 \mathrm{~g})=2350 \mathrm{mg}$ \\
\hline $\begin{array}{l}\text { Semente de linhaça } \\
\text { triturada }\end{array}$ & 1597 & - & - & - & 1 colher de sopa $(7,0 \mathrm{~g})=1597 \mathrm{mg}$ \\
\hline Semente de chia & 1808 & - & - & - & 1 colher de sopa $(10,3 \mathrm{~g})=1808 \mathrm{mg}$ \\
\hline Óleo de linhaça & 7249 & - & - & - & 1 colher de sopa $(13,6 \mathrm{~g})=7249 \mathrm{mg}$ \\
\hline \multicolumn{6}{|l|}{ Origem animal $*$} \\
\hline Óleo de Salmão & - & 586 & 820 & 183 & 1 colher de chá $(4,5 \mathrm{~g})=1589 \mathrm{mg}$ \\
\hline Óleo de Menhaden & - & 593 & 385 & 288 & 1 colher de chá $(4,5 \mathrm{~g})=1266 \mathrm{mg}$ \\
\hline $\begin{array}{l}\text { Óleo de Fígado de } \\
\text { Bacalhau }\end{array}$ & - & 310 & 494 & 84 & 1 colher de chá $(4,5 \mathrm{~g})=888 \mathrm{mg}$ \\
\hline Óleo de Arenque & - & 282 & 189 & 62 & 1 colher de chá $(4,5 \mathrm{~g})=533 \mathrm{mg}$ \\
\hline Salmão & - & 680 & 1457 & 113 & 1 filé de $100 \mathrm{~g}=2260 \mathrm{mg}$ \\
\hline Arenque & - & 909 & 1105 & 203 & 1 filé de $100 \mathrm{~g}=2217 \mathrm{mg}$ \\
\hline Cavalinha & - & 504 & 699 & 219 & 1 filé de $100 \mathrm{~g}=1422 \mathrm{mg}$ \\
\hline Truta & - & 259 & 667 & 434 & 1 filé de $100 \mathrm{~g}=1360 \mathrm{mg}$ \\
\hline Atum & - & 91 & 237 & 17 & 1 filé de $100 \mathrm{~g}=345 \mathrm{mg}$ \\
\hline Sardinha $* *$ & - & 20 & 107 & 183 & 1 filé de $100 \mathrm{~g}=310 \mathrm{mg}$ \\
\hline Hadock & - & 76 & 162 & 24 & 1 filé de $100 \mathrm{~g}=262 \mathrm{mg}$ \\
\hline
\end{tabular}

Quadro 2. Alimentos fontes de n-3 PUFA, composição dos ácidos graxos, ácido graxo predominante (em negrito) e teor total de acordo com uma porção usual de consumo. 


\section{SUPLEMENTOS}

Os suplementos de n-3 PUFA mais comuns disponíveis no mercado brasileiro são constituídos de óleo de peixe e de linhaça. Formulações com óleo de Chia e Microalga são comercializadas internacionalmente. A emulsão de óleo de peixe em sachê é vendida nos EUA. O US Food and Drug Administration (FDA) aprovou uma única formulação com alta concentração de ésteres de n-3 PUFA que requer prescrição médica e é destinada a indivíduos com concentrações séricas de triglicérides $\geq 500$ $\mathrm{mg} / \mathrm{dL}{ }^{[7]}$ (Quadro 3).

\section{LINHAÇA}

Em sua composição, a massa de linhaça possui de 32 a $45 \%$ de óleo, do qual 51 a 55\% é ALA. Estudo que avaliou o efeito de linhaça na forma de semente ou outro derivado apontaram redução de $0 \mathrm{a}$ $11 \%$ e 0 a $18 \%$ nos níveis séricos de Colesterol Total (CT) e de Lipoproteína de Baixa Densidade (LDL-c), respectivamente ${ }^{[8]}$. É importante considerar que, além do ALA, a semente da linhaça contém lignanas [?]. A principal delas é a Secoisolariciresinol dighycoside (SDG), que precisa ser convertida pela microbiota intestinal humana em enterodiol e enterolactona, suas formas biologicamente ativas [10]. Possivelmente, a supressão da aterosclerose associada ao consumo da semente de linhaça resulta do conteúdo de lignanas e não do ALA [10], porém há controvérsias [11]. Em relação ao perfil lipídico, Pan et al. [12], em uma meta-análise, observaram que a semente de linhaça está associada com significante redução nas concentrações de colesterol total e de LDL-c, sem efeito nas concentrações de triglicérides. Os autores associaram essa redução com o alto teor de fibras solúveis existente na semente, pois não foram observadas alterações nas intervenções com óleo de linhaça.

Quanto ao efeito anti-inflamatório, os resultados da literatura também não são consistentes [8,13]. Intervenção com doses de óleo de linhaça superiores a 14 g estão associadas à redução nas concentrações de marcadores inflamatórios. Tal consumo equivale a duas colheres de sopa ou 49 cápsulas de $500 \mathrm{mg}$ de óleo de linhaça por dia.
Segundo Ulbricht et al. [14], as evidências sobre a eficácia da semente de chia são insuficientes, considerando a escassez de estudos que avaliaram o papel cardioprotetor dessa semente. Nieman et al. [15] não observaram mudanças significativas no peso corporal e em marcadores de risco, incluindo inflamatórios, em adultos com sobrepeso após o consumo de $25 \mathrm{~g}$ de semente de chia/dia por 12 semanas. Em contrapartida, Vuksan et al. [16] observaram redução significativa de pressão arterial, proteína $\mathrm{C}$ reativa e fator de von Willebrand em indivíduos com diabetes tipo 2 após consumo de $37 \mathrm{~g}$ da semente/dia. Em outro estudo, foi avaliado o efeito de pão contendo $0,7,15$ ou $24 \mathrm{~g}$ de semente de chia na glicemia pós-prandial e na saciedade em adultos. Foi observada redução na glicose pós-prandial obedecendo a um padrão dose-resposta de acordo com cada proporção de chia incorporada ao pão. $\mathrm{O}$ aumento na saciedade foi descrito em todos os grupos (7, 15 ou $24 \mathrm{~g}$ ) quando comparados ao controle (pão isento de chia) [17]. Não foram localizados estudos que descrevem o efeito de intervenções com óleo de chia.

\section{MICROALGA}

As algas marinhas podem ser consideradas uma fonte alternativa de n-3 PUFA em relação aos peixes, uma vez que o óleo extraído é concentrado e, provavelmente, contém menor teor de contaminantes. O óleo de alga tem sido utilizado na produção de ovos ricos em n-3 PUFA, na aquicultura para aumentar a concentração desses ácidos em peixes e, também, na produção de suplementos para consumo humano [18].

Os resultados de três ensaios clínicos conduzidos com vegetarianos saudáveis apontam redução nas concentrações de triglicérides, variando de $17-23 \%$, após suplementações com 940-2140 mg de DHA/dia durante 6-8 semanas. Em indivíduos onívoros saudáveis, os resultados de cinco ensaios clínicos mostram redução de 2-26\%, com doses de $700-6000 \mathrm{mg} /$ dia por 4-12 semanas. Para populações com diferentes alterações no perfil lipídico incluídas em cinco ensaios clínicos, 1000-3000 mg de DHA/dia associou-se com redução de $18-24 \%$ nas concentrações de triglicérides após 6-12 semanas. A meta-análise desses resultados indica que doses de 1000-2000 mg de DHA/dia reduzem significantemente as concentrações de triglicérides em proporções de $15-20 \%$ [19]. Sendo assim, essa ingestão equivaleria à suplementação com 4 a 10 cápsulas/dia, dependendo da dose.

\section{CHIA}


Em relação aos demais parâmetros cardiovasculares, observam-se aumento da concentração de HDL-c, redução modesta na pressão arterial e na frequência de pulso, bem como promoção do relaxamento endotelial. Além desses efeitos, o suplemento com óleo de microalga, diferentemente do óleo de peixe, raramente causa algum desconforto como refluxo e/ou gosto de peixe, o que é importante para a adesão ao tratamento, além de ser apropriado para vegetarianos e veganos ${ }^{[19]}$.

\begin{tabular}{|c|c|c|c|c|c|c|}
\hline \multirow{2}{*}{$\begin{array}{l}\text { Suplementos/ } \\
\text { Formulações }\end{array}$} & \multirow{2}{*}{ Características } & \multicolumn{5}{|c|}{ Composição (mg) } \\
\hline & & ALA $^{1}$ & $\mathrm{EPA}^{2}$ & $\mathrm{DHA}^{3}$ & Outros & Total \\
\hline \multicolumn{7}{|l|}{ Origem vegetal } \\
\hline Óleo de Linhaça & $\begin{array}{l}\text { Cápsula gelatinosa } \\
\qquad(500 \mathrm{mg})\end{array}$ & 285 & - & - & - & 285 \\
\hline Óleo de Chia & $\begin{array}{l}\text { Cápsula vegana } \\
\text { (500 mg) }\end{array}$ & 285 & - & - & - & 285 \\
\hline $\begin{array}{l}\text { Óleo de Microalga (com } \\
\text { licopeno) }\end{array}$ & $\begin{array}{l}\text { Líquido aromatizado } \\
\text { (14 gotas) }\end{array}$ & - & - & 200 & - & 200 \\
\hline Óleo de Microalga & $\begin{array}{l}\text { Cápsula vegana } \\
\text { (500 mg) }\end{array}$ & - & - & 200 & - & 200 \\
\hline Óleo de Microalga & $\begin{array}{l}\text { Cápsula vegana } \\
\text { (800 mg) }\end{array}$ & - & 10 & 300 & - & 310 \\
\hline \multicolumn{7}{|l|}{ Origem animal } \\
\hline Óleo de Peixe & $\begin{array}{l}\text { Cápsula gelatinosa } \\
\text { (1000 mg) }\end{array}$ & - & 180 & 120 & - & 300 \\
\hline $\begin{array}{l}\text { Óleo de Peixe com alta } \\
\text { concentração de DHA }\end{array}$ & $\begin{array}{l}\text { Cápsula gelatinosa } \\
\quad(1000 \mathrm{mg})\end{array}$ & - & 100 & 500 & - & 600 \\
\hline $\begin{array}{l}\text { Óleo de Peixe com alta } \\
\text { concentração de EPA }\end{array}$ & $\begin{array}{l}\text { Cápsula gelatinosa } \\
\quad(1000 \mathrm{mg})\end{array}$ & - & 540 & 100 & - & 640 \\
\hline $\begin{array}{l}\text { Emulsão de Óleo de } \\
\text { Peixe }\end{array}$ & $\begin{array}{l}\text { Sachês contendo a } \\
\text { emulsão aromatizada } \\
\text { (2500 mg) }\end{array}$ & - & 350 & 230 & - & 650 \\
\hline Éster de Ômega-3 & $\begin{array}{l}\text { Cápsula gelatinosa } \\
\quad(1000 \mathrm{mg})\end{array}$ & - & 465 & 375 & - & 840 \\
\hline
\end{tabular}

${ }^{1} \mathrm{ALA}=$ ácido alfa-linolênico; ${ }^{2} \mathrm{EPA}=$ ácido eicosapentaenoico; ${ }^{3} \mathrm{DHA}$ = ácido docosahexaenóico.

Quadro 3. Suplementos e formulações de n-3 PUFA, características, composição e ácido graxo predominante (em negrito). 


\section{PEIXE}

Estudos experimentais, observacionais e revisões, que tratam do papel do n-3 PUFA presentes em peixes e em óleo de peixe são abundantes e seu papel cardioprotetor está bem discutido na literatura. Segundo Wang et al. [20], os efeitos atribuídos ao óleo de peixe são mais evidentes na prevenção secundária, isto é, em populações com alguma alteração cardiovascular prévia. Em relação à prevenção primária, as evidências são controversas [20,21].

De acordo com a American Heart Association (AHA), estudos prospectivos de prevenção secundária demonstram que doses de 500 a 1800 mg de EPA+DHA, provenientes de suplementos ou consumo de peixe, reduzem a mortalidade em geral e por eventos cardiovasculares. Para indivíduos com doença cardiovascular documentada, aproximadamente $1000 \mathrm{mg}$ de EPA+DHA, preferencialmente derivados do consumo de peixes ricos em gordura, são recomendados. Para redução de $25-45 \%$ nas concentrações de triglicérides, a recomendação é de 2000-4000 mg de EPA+DHA provenientes de cápsulas de óleo de peixe ou formulação de éster de ômega-3 [2,7]. Sendo assim, altas doses, de 7 a 13 cápsulas de óleo de peixe tradicional/dia seriam necessárias.

Apesar da ausência de fortes evidências em relação à prevenção primária associada ao consumo de n-3 PUFA, a AHA recomenda, para indivíduos sem doença cardiovascular diagnosticada, o consumo de peixe duas vezes por semana, de preferência ricos em gordura, além de outras fontes de ALA, como a linhaça [2]. Segundo Musa-Veloso et al. [22], comitês internacionais recomendam a ingestão de 200-500 $\mathrm{mg} /$ dia de n-3 PUFA para populações saudáveis. Os autores apontam que tais doses estão associadas a reduções nas concentrações de triglicérides variando de $-3,1 \%$ (IC95\%: $-5,9$ a $-0,31 \%$ ) a $-7,2 \%$ (IC95\%:$11,6$ a $-2.5 \%)$. Esses resultados podem apresentar benefícios do ponto de vista da saúde coletiva se somados a demais esforços na manutenção de concentrações adequadas de triglicérides. Tal consumo equivaleria a cerca de uma a duas cápsulas de óleo de peixe tradicional/dia.

\section{FONTES DE N-3 PUFA E SUSTENTABILIDADE AMBIENTAL}

Equacionar as recomendações, as evidências científicas em relação aos benefícios do n-3 PUFA para a saúde humana e os impactos ambientais da exploração dos estoques pesqueiros naturais, constituise em um grande desafio. As reservas de peixe têm sofrido rápido declínio nos últimos 50 anos. Algumas espécies comerciais apresentam-se reduzidas a $10 \%$ da sua população inicial e mais de 100 espécies já foram extintas [23]. Das reservas de peixes marinhos monitoradas pela Food and Agriculture Organization of the United Nations (FAO), 71\% já foram muito exploradas e essas espécies estão em seu limite de reposição natural [24]. Esse caráter essencialmente extrativista da atividade tem sido motivo de preocupação para os gestores e especialistas do setor em todo o mundo. Como recursos naturais, os estoques pesqueiros seguem regras biológicas de reprodução, pois cada estoque é multiespecífico e a captura direcionada para uma determinada espécie sempre acaba atuando sobre as demais, o que torna bastante complexa a tarefa de manter o equilíbrio dos ecossistemas marinhos [25]. A pressão sobre as reservas se intensifica ao mesmo tempo em que há aumento das recomendações para o consumo de peixe e de seu óleo [23,26]. Para atender à demanda, a produção mundial de peixe aumentou de aproximadamente 20 milhões de toneladas, em 1950, para mais de 140 milhões, em 2006 [24].

Outro ponto importante a ser discutido é o crescimento da aquicultura. Esse setor se desenvolveu mais rapidamente que a produção de outras carnes. Além disso, tem se tornado muito importante na provisão de peixe para consumo humano. Porém, a aquicultura envolve a utilização de pequenos peixes selvagens, como a anchoveta, para a produção de ração. Atualmente, cerca de um quarto dos peixes capturados das reservas naturais são utilizados para a produção de ração. A proporção de n-3 PUFA na ração depende do perfil alimentar dos peixes, ou seja, se são herbívoros, onívoros ou carnívoros. Os peixes carnívoros, como o salmão, necessitam de ração com maior concentração de n-3 PUFA quando comparados aos herbívoros [23,27], o que pressiona ainda mais as reservas naturais de pequenos peixes. Assim, a não ser que haja uma dissociação entre a exploração das reservas de peixes e a aquicultura, especialmente por meio do desenvolvimento de fontes alternativas que mantenham o valor para a saúde humana dos peixes produzidos pela aquicultura, provavelmente essa alternativa não será suficiente para manter o fornecimento sustentável para o consumo humano. 
Deve-se considerar também o monitoramento da concentração de xenobióticos, como as dioxinas, policlorinato bifenil (PCBs) e mercúrio [2]. A concentração dessas substâncias nas águas dos oceanos é reduzida, porém está aumentada na cadeia alimentar marinha, principalmente nos peixes predadores de grande porte. Tais substâncias também podem estar presentes no óleo de pequenos peixes selvagens utilizados na aquicultura. Uma possível solução seria a mudança na composição da ração com substituição do óleo de pequenos peixes por óleo de microalgas. Isso possibilitaria uma contaminação mínima, além das algas constituírem fonte primária de n-3 PUFA na cadeia alimentar marinha [26,27], o que poderia contribuir para a conservação da biodiversidade e preservação das reservas de peixes selvagens.

A exploração das reservas de peixes também tem implicações na segurança alimentar e nutricional de pessoas que vivem nas regiões costeiras de países com menor desenvolvimento econômico. Como a demanda pelo consumo de peixes, especialmente os ricos em n-3 PUFA, em países desenvolvidos vem crescendo por conta das recomendações associadas aos benefícios à saúde, essa dinâmica propicia o aumento de iniquidades entre e dentre as populações. Dessa forma, a competição pelo peixe tende a aumentar a vulnerabilidade social, econômica e nutricional de países com menor desenvolvimento. Enquanto isso, populações de países mais ricos e com melhores condições sociais e econômicas tendem a manter alto nível de saúde e segurança alimentar e nutricional [28].

\section{CONSIDERAÇÕES FINAIS}

Apesar dos benefícios relacionados à ingestão de n-3 PUFA estarem bem discutidos na literatura científica, há evidências de impossibilidade ambiental de atender as demandas de consumo de peixes fontes desse ácido graxo. Assim, a utilização de óleo de microalgas para a aquicultura e para o consumo humano pode ser uma alternativa, mas deve-se avaliar seu uso com responsabilidade para que não ocorram desequilíbrios ambientais.

\section{REFERÊNCIAS}

[1] Institute of Medicine (IOM). Dietary fats: total fat and fatty acids. In: Dietary reference intakes for energy, carbohydrate, fiber, fat, fatty acids, cholesterol, protein, and amino acids. Washington, DC: National Academy Press; 2002. p. $422-541$.

[2] Kris-Etherton PM, William SH, Appel LJ. Fish consumption, fish oil, n-3 fatty acids, and cardiovascular disease. Circulation. 2002;106(21):2747-57.

[3] Lee JH, O'Keefe JH, Lavie CJ, Harris WS. N-3 fatty acids: cardiovascular benefits, sources and sustainability. Nat Rev Cardiol. 2009;6(12):753-8.

[4] Simopoulos AP. The omega- $6 / \mathrm{n}-3$ fatty acid ratio, genetic variation, and cardiovascular disease. Asia Pac J Clin Nutr. 2008;17(Suppl 1):131-4.

[5] United States Department of Agriculture, Agricultural Research Service [homepage] 2008. USDA National Nutrient Database for Standard Reference, Release 21. Nutrient Data Laboratory [cited 2012 jun 28]. Available from: http://www.ars.usda.gov/ba/bhnrc/ndl

[6] Luzia LA, Sampaio GR, Castellucci C, Torres E. The influence of season on the lipids profiles of five commercially important species of Brazilian fishes. Food Chemistry. 2003;83(1):93-7.

[7] Sadovsky R, Kris-Etherton P. Prescription omega-3-acid ethyl esters for the treatment of very high triglycerides. Postgrad Med. 2009;121(4):145-53.

[8] Bloedon LT, Szapary PO. Flaxseed and cardiovascular risk. Nutr Rev. 2004;62(1):18-27.

[9] Prasad K. Flaxseed and cardiovascular health. J Cardiovasc Pharmacol 2009;54(5):369-77.

[10] Thompson LU, Robb P, Serraino M, Cheung F. Mammalian lignan production from various foods. Nutr Cancer. 1991;16(1):43-52.

[11] Peterson J, Dwyer J, Adlercreutz H, Scalbert A, Jacques P, McCullough ML. Dietary lignans: physiology and potential for cardiovascular disease risk reduction. Nutr Rev. 2010;68(10):571-603.

[12] Pan A, Yu D, Demark-Wahnefried W, Franco OH, Lin $\mathrm{X}$. Meta-analysis of the effects of flaxseed interventions on blood lipids. Am J Clin Nutr. 2009;90(2):288-97.

[13] Basch E, Bent S, Collins J, Dacey C, Hammerness P, Harrison M, et al. Flax and flaxseed oil (linum usitatissimum): a review by the Natural Standard Research Collaboration. J Soc Integr Oncol. 2007;5(3):92-105. 
[14] Ulbricht C, Chao W, Nummy K, Rusie E, TanguayColucci S, Iannuzzi CM, et al. Chia (salvia hispanica): a systematic review by the Natural Standard Research Collaboration. Rev Recent Clin Trials. 2009;4(3):168-74.

[15] Nieman DC, Cayea EJ, Austin MD, Henson DA, McAnulty SR, Jin F. Chia seed does not promote weight loss or alter disease risk factors in overweight adults. Nutr Res. 2009;29(6):414-8.

[16] Vuksan V, Whitham D, Sievenpiper JL, Jenkins AL, Rogovik AL, Bazinet RP, et al. Supplementation of conventional therapy with the novel grain salba (salvia bispanica l.) improves major and emerging cardiovascular risk factors in type 2 diabetes: results of a randomized controlled trial. Diabetes Care. 2007;30(11):2804-10.

[17] Vuksan V, Jenkins AL, Dias AG, Lee AS, Jovanovski E, Rogovik AL, et al. Reduction in postprandial glucose excursion and prolongation of satiety: possible explanation of the long-term effects of whole grain salba (salvia hispanica l.). Eur J Clin Nutr. 2010;64(4):436-8.

[18] Colombo ML, Risè P, Giavarini F, De Angelis L, Galli C, Bolis CL. Marine macroalgae as sources of polyunsaturated fatty acids. Plant Foods Hum Nutr. 2006;61(2):67-72.

[19] Ryan AS, Keske MA, Hoffman JP, Nelson EB. Clinical overview of algal-docosahexaenoic acid: effects on triglyceride levels and other cardiovascular risk factors. Am J Ther. 2009;16(2):183-92.

[20] Wang C, Harris WS, Chung M, Lichtenstein AH, Balk EM, Kupelnick B, et al. N-3 Fatty acids from fish or fish-oil supplements, but not alfa-linolenic acid, benefit cardiovascular disease outcomes in primary- and secondaryprevention studies: a systematic review. Am J Clin Nutr. 2006;84(1):5-17.
[21] Saravanan P, Davidson NC, Schmidt EB, Calder PC. Cardiovascular effects of marine omega-3 fatty acids. Lancet. 2010;376(9740):540-50.

[22] Musa-Veloso K, Binns MA, Kocenas AC, Poon T, Elliot JA, Rice $\mathrm{H}$, et al. Long-chain omega-3 fatty acids eicosapentaenoic acid and docosahexaenoic acid dosedependently reduce fasting serum triglycerides. Nutr Rev. 2010;68(3):155-67.

[23] Lee JH, O'Keefe JH, Lavie CJ, Harris WS. Omega-3 fatty acids: cardiovascular benefits, sources and sustainability. Nat Rev Cardiol. 2009;6(12):753-8.

[24] Food and Agriculture Organization of the United Nations. The state of world fisheries and aquaculture 2008. FAO Fisheries and Aquaculture Department. Rome, 2009.

[25] Klippel S, Peres M. Pesca, aquicultura e meio ambiente. Instituto Igaré e Centro de Pesquisa e Gestão dos Recursos Pesqueiros Estuarinos e Lagunares (Ceperg) 2004 [acesso em 28 jun 2012]. Disponível em: http://br.geocities.com/institutoigare/fepamdebate/aprese ntacao.pdf

[26] Canon D. From fish oil to microalgae oil... A win-win shift for humans and our habitat. Explore (NY). 2009; 5(5):299-303.

[27] Miller MR, Nichols PD, Carter CG. N-3 oil sources for use in aquaculture - alternatives to the unsustainable harvest of wild fish. Nutr Res Rev. 2008;21(2):85-96.

[28] Brunner EJ, Jones PJS, Friel S, Bartley M. Fish, human health and marine ecosystem health: polices in collision. Int J Epidemiol. 2009;38(1):93-100. 